\title{
Water, Energy, and Carbon with Artificial Neural Networks (WECANN): A statistically-based estimate of global surface turbulent fluxes using solar-induced fluorescence
}

Seyed Hamed Alemohammad, Bin Fang, Alexandra G. Konings, Julia K. Green, Jana Kolassa, Catherine Prigent, Filipe Aires, Diego Miralles, Pierre Gentine

\section{Supplementary Material}




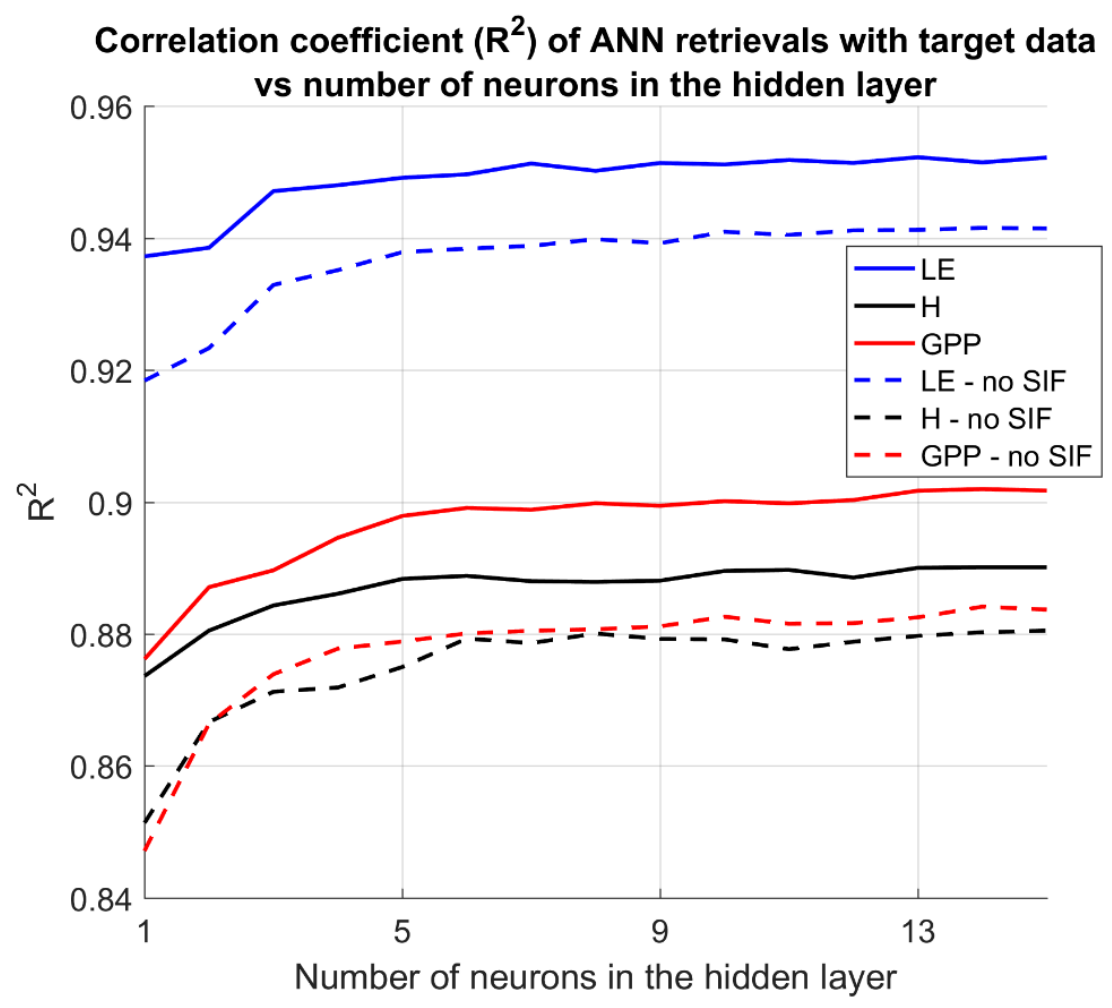

Figure S1: Correlation coefficients $\left(R^{2}\right)$ between ANN retrievals of LE, $H$, and GPP and target data as a function of number of neurons in hidden layer. Solid lines show $R^{2}$ for retrievals that include SIF as an input in ANN, and dashed lines show $R^{2}$ for retrievals without SIF as an input. 

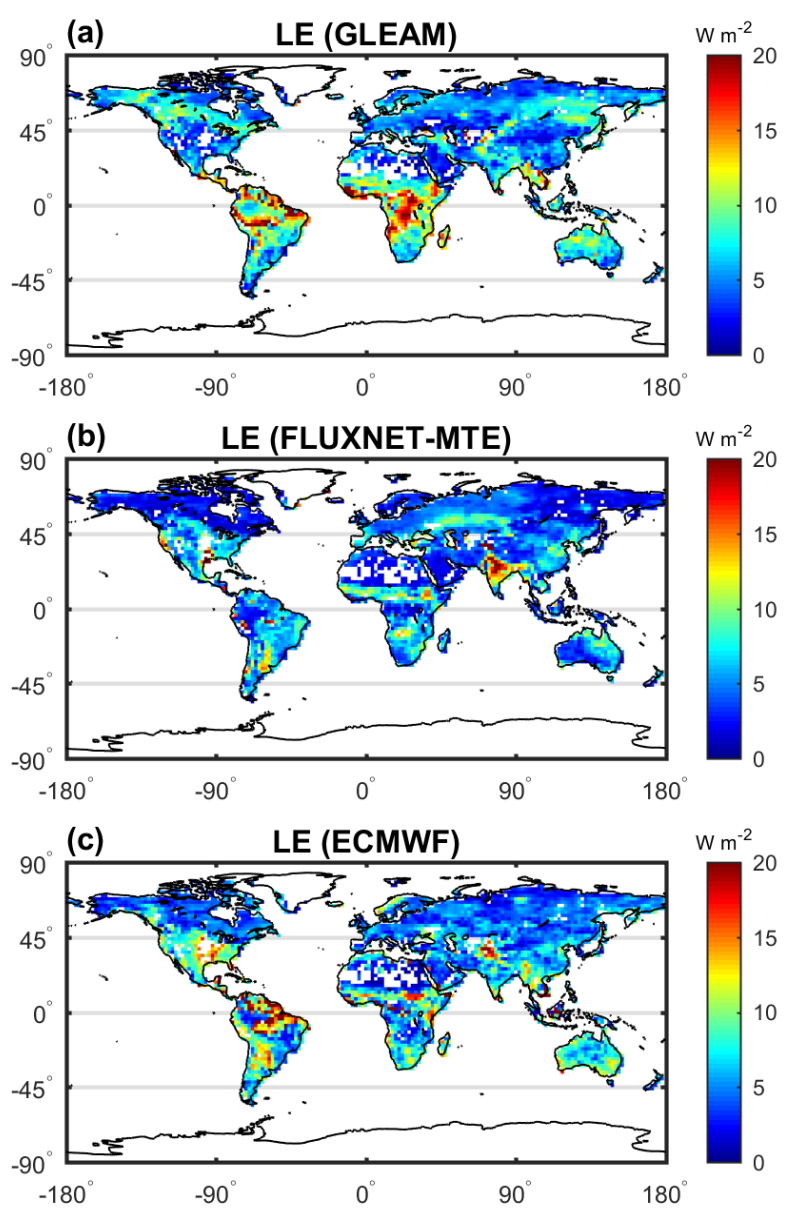

Figure S2: RMSE of LE for each of the three training datasets estimated by Triple Collocation (2008-2010). 

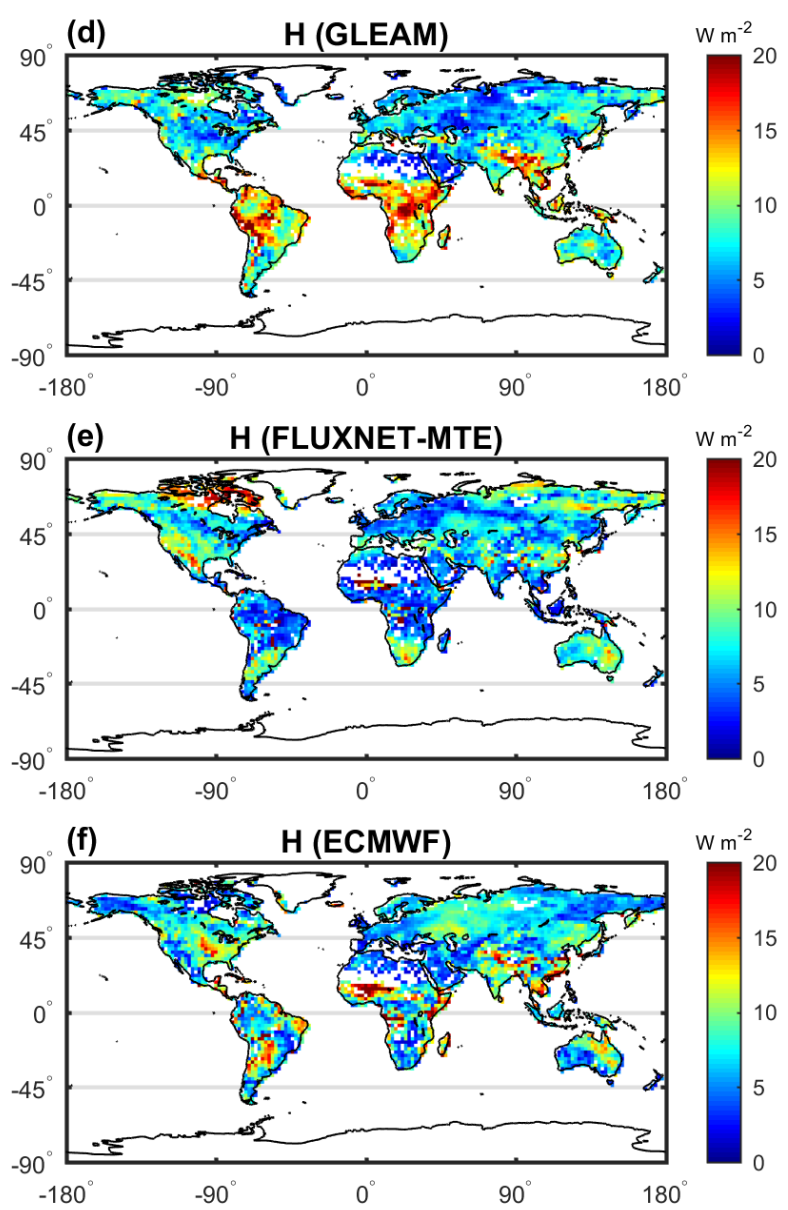

Figure S3: Similar to Figure S1 for $H$. 

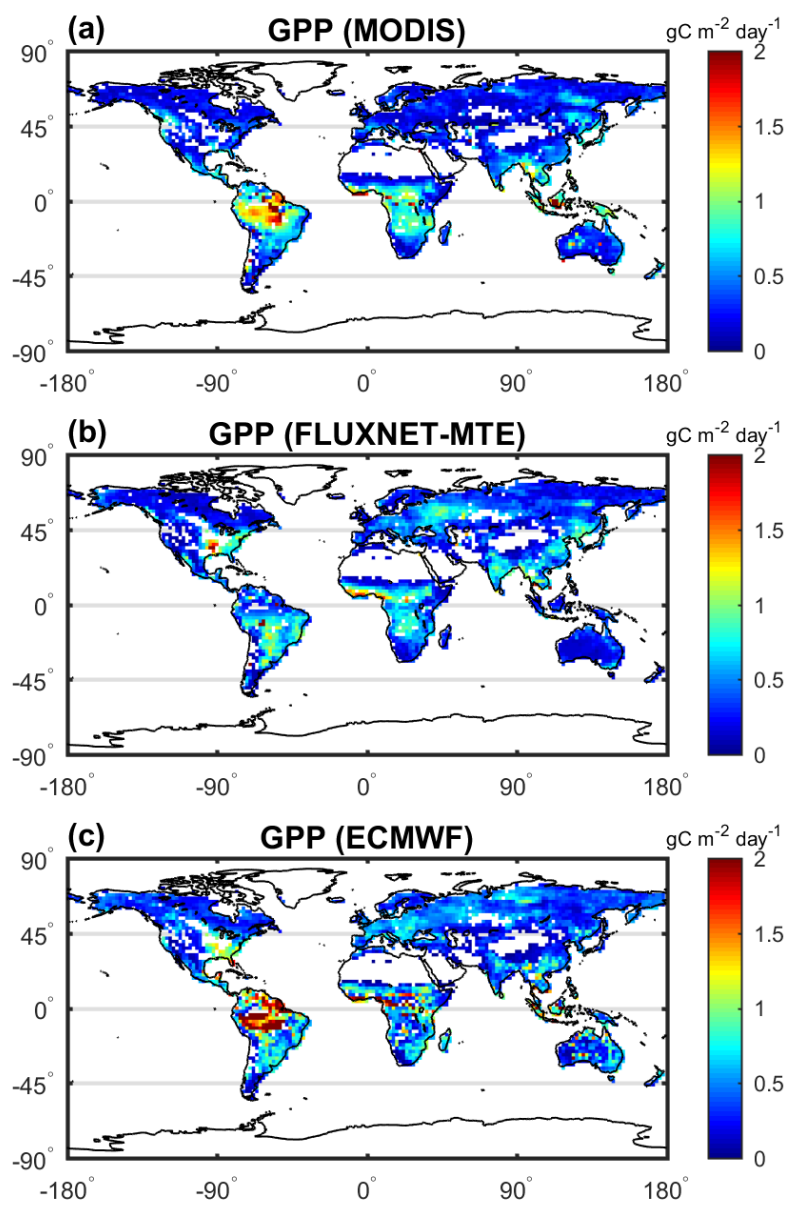

Figure S4: Similar to Figure S1 for GPP. 